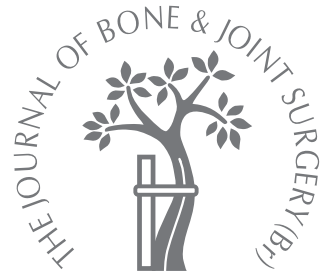

B. J. A. Lankester, H. L. Cottam, V. Pinskerova, J. D. J. Eldridge, M. A. R. Freeman

From the Bristol Royal Infirmary, Bristol, England

\title{
Variation in the anatomy of the tibial plateau
}

\author{
A POSSIBLE FACTOR IN THE DEVELOPMENT OF ANTEROMEDIAL \\ OSTEOARTHRITIS OF THE KNEE
}

B. J. A. Lankester, FRCS(Tr \& Orth), Consultant Orthopaedic Surgeon

Yeovil District Hospital, Higher Kingston, Yeovil BA21 4AT, UK.

H. L. Cottam, MRCS

Specialist Registrar in Trauma and Orthopaedics

Royal Sussex County Hospital,

Eastern Road, Brighton BN2

$5 B E, U K$.

- V. Pinskerova, MD, PhD,

Associate Professor

= M. A. R. Freeman, MD, FRCS,

Visiting Professo

1st Orthopaedic Clinic

Charles University, 1st

Orthopaedic Clinic, Charles

University, V Uvalu 84, 15006

Prague 5, Czech Republic.

I. J. D. J. Eldridge, FRCS(Tr \&

Orth), Consultant Orthopaedic

Surgeon

Bristol Royal Infirmary,

Marlborough Street, Bristol

BS2 8HW, UK.

Correspondence should be sent to Mr B. J. A. Lankester; email: ben.lankester@ydh.nhs.uk

(ㅇ)2008 British Editorial Society of Bone and Joint Surgery doi:10.1302/0301-620X.90B3. $19898 \$ 2.00$

$J$ Bone Joint Surg $[\mathrm{Br}]$ 2008;90-B:330-3.

Received 2 July 2007;

Accepted 18 October 2007

\begin{abstract}
From a search of MRI reports on knees, 20 patients were identified with evidence of early anteromedial osteoarthritis without any erosion of bone and a control group of patients had an acute rupture of the anterior cruciate ligament. The angle formed between the extension and flexion facets of the tibia, which is known as the extension facet angle, was measured on a sagittal image at the middle of the medial femoral condyle.

The mean extension facet angle in the control group was $14^{\circ}\left(3^{\circ}\right.$ to $\left.25^{\circ}\right)$ and was unrelated to age (Spearman's rank coefficient, $p=0.30, r=0.13$ ). The mean extension facet angle in individuals with MRI evidence of early anteromedial osteoarthritis was $19^{\circ}\left(13^{\circ}\right.$ to $26^{\circ}$, SD $\left.4^{\circ}\right)$. This difference was significant (Mann-Whitney $U$ test, $p<0.001$ ).

A wide variation in the extension facet angle was found in the normal control knees and an association between an increased extension facet angle and MRI evidence of early anteromedial osteoarthritis. Although a causal link has not been demonstrated, we postulate that a steeper extension facet angle might increase the duration of loading on the extension facet during the stance phase of gait, and that this might initiate failure of the articular cartilage.
\end{abstract}

Osteoarthritis (OA) of the knee most commonly affects the medial tibiofemoral articulation. ${ }^{1}$ The location of the tibial lesion within the medial compartment was first described in detail by White, Ludkowski and Goodfellow, ${ }^{2}$ who examined specimens retrieved after medial unicompartmental replacement undertaken in patients in whom the anterior cruciate ligament (ACL) was intact, and correlated the findings with sagittal plane radiological appearances. They found that the erosion was limited to the anterior part of the plateau, and coined the term 'anteromedial osteoarthritis of the knee' to describe a distinct clinicopathological entity.

Keyes et $\mathrm{al}^{3}$ studied medial compartmental OA of the knee and separated those patients with anterocentral erosion from those who displayed a more posterior erosion, which was invariably associated with degenerative rupture of the ACL. These findings were confirmed by Harman et $\mathrm{al}^{4}$ and Moschella et al, ${ }^{5}$ who also demonstrated that in ACL-deficient varus knees, the area of tibial erosion was greater and extended more posteriorly than in ACL-intact knees.

Detailed descriptions of the shape of the medial tibial condyle were made by 19 th century German anatomists. ${ }^{6}$ More recent cadaver studies and MRI analysis ${ }^{7}$ have further determined the normal sagittal anatomy of the medial tibial plateau. This is composed of two relatively flat facets, an anterior upwardsloping extension facet, and a posterior, roughly horizontal surface or flexion facet that lies predominantly under the posterior horn of the medial meniscus (Fig. 1). The area of the tibia in which anteromedial OA is said to begin corresponds to the base of the extension facet.

MRI studies in unloaded cadaver knees, of the relative movements of the femoral and tibial condyles show that the medial femoral condyle articulates with the horizontal flexion facet of the tibia, with a relatively constant contact area from $30^{\circ}$ to $120^{\circ}$ flexion. As the knee extends from $30^{\circ}$, contact with the medial femoral condyle shifts forward to the extension facet. ${ }^{7}$ From $10^{\circ}$ to full extension in humans (and bears, but not in other quadruped mammals in which the knee does not extend beyond $20^{\circ}$ ), the medial femoral condyle contacts only the extension facet, losing contact with the flexion facet and lifting posteriorly by $2 \mathrm{~mm}$ to $3 \mathrm{~mm}$ as it does so. ${ }^{8}$

In the MRI studies described, ${ }^{7,8}$ the angle between the extension facet and the flexion facet (which we termed the extension facet angle) was measured on scans of six cadaver 


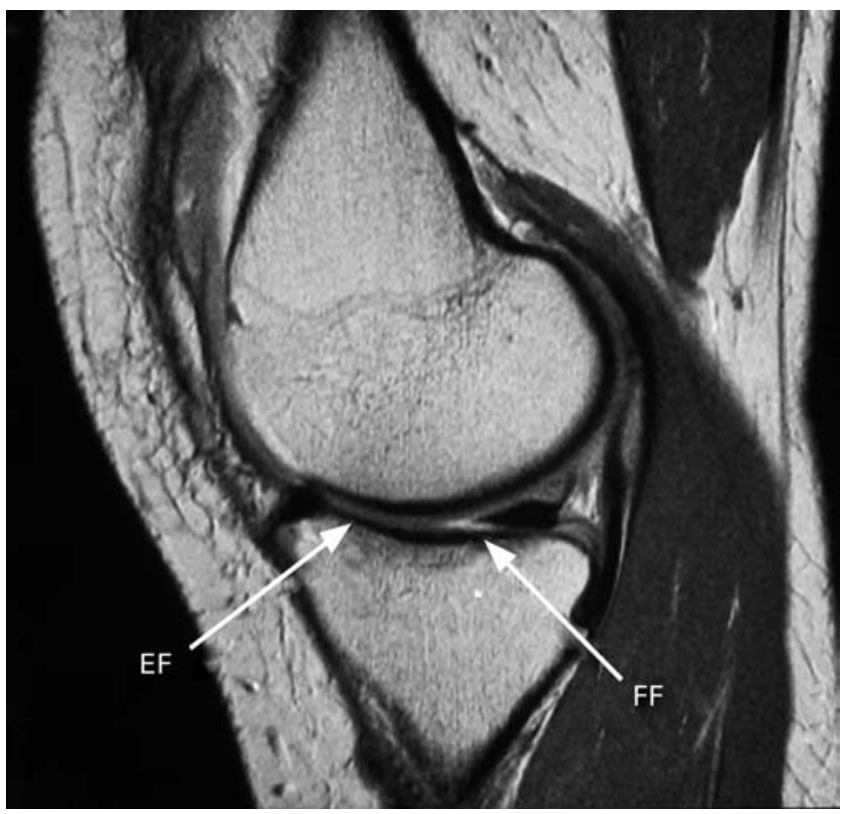

Fig. 1

Extension facet (EF) and flexion facet (FF) on a sagittal MRI slice through the midpoint of the medial femoral condyle.

knees, finding a mean extension facet angle of $11^{\circ}\left(8^{\circ}\right.$ to $\left.15^{\circ}\right)$. The anatomical coincidence between the apparent site of origin of anteromedial OA and the base of the extension facet led us to investigate the extension facet angle in a larger number of normal living knees, and to compare this with the extension facet angle in a group of patients with MRI evidence of isolated early anteromedial OA.

\section{Patients and Methods}

Hundreds of knee MRI reports prepared by a consultant musculoskeletal radiologist were searched to identify two specific groups of patients: those with an acutely ruptured ACL without any other reported pathology (64 patients; 46 men and 18 women) and those with MRI evidence of early isolated medial compartment OA, which had been reported as either early medial compartment degeneration or chondral changes, but with no bone-to-bone contact, no ACL rupture and no meniscal tear (20 patients; 11 men and nine women). The subjects' ages and the date of the scans were recorded, but no details of symptoms or clinical diagnosis were obtained.

The scans were performed using a dedicated knee coil on a 1.5 Tesla scanner (Symphony Maestro, Siemens Medical Systems, Camberley, United Kingdom). The sequences examined were the sagittal turbo spin echo $\mathrm{T}_{2}$-weighted scans. The slices were orientated perpendicular to the line connecting the posterior femoral condylar cortices. A slice that passed through the centre of the medial femoral condyle, which was generally $12 \mathrm{~mm}$ from the medial tibial cortex was chosen (Fig. 1).

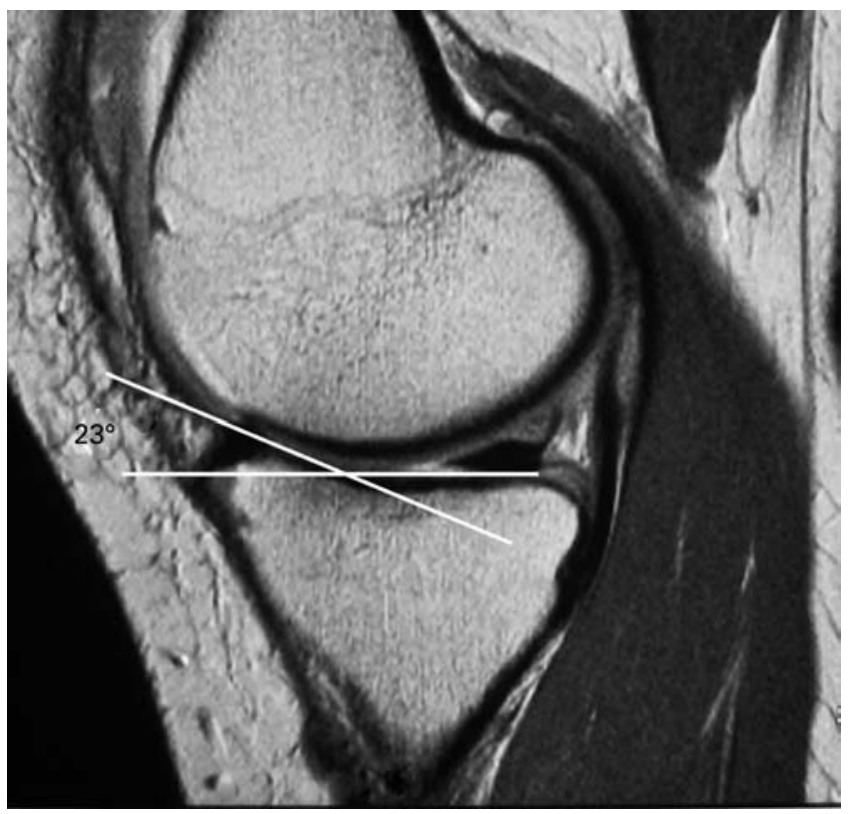

Fig. 2

MRI showing measurement of the extension facet angle.

The images were loaded on to a Leonardo workstation (Siemens Medical Systems) using Syngo post-processing software (Siemans Medical Systems) which allows direct measurement of angles from lines drawn at the junction of cartilage and subchondral bone, along the roughly horizontal flexion facet and the upwardly sloping extension facet. The angle between them is then calculated automatically (Fig. 2). The measurement was made on two separate occasions by one observer (BJAL) with the images presented in random order and blinded to diagnosis, and also by a second observer (HLC) to establish intra- and interobserver reproducibility.

Statistical analysis. Guidance on study design and assistance with statistical analysis was obtained from a medical statistician. The intra-class correlation coefficient was used to assess inter- and intra-observer reliability. For descriptive analysis, the mean, standard deviation (SD) and range of values for each group were calculated. The relationship between age and extension facet angle was examined using Spearman's rank coefficient. Comparisons between the groups were made using the Mann Whitney U test. Statistical significance was set at $\mathrm{p}<0.05$.

\section{Results}

The method of measurement was found to be both reproducible and reliable. The intra-class correlation coefficient was 0.918 for repeated measurements by one observer and 0.922 for measurements made by different observers, indicating good reproducibility. On one image, a difference of $8^{\circ}$ was recorded by different observers (BJAL, 


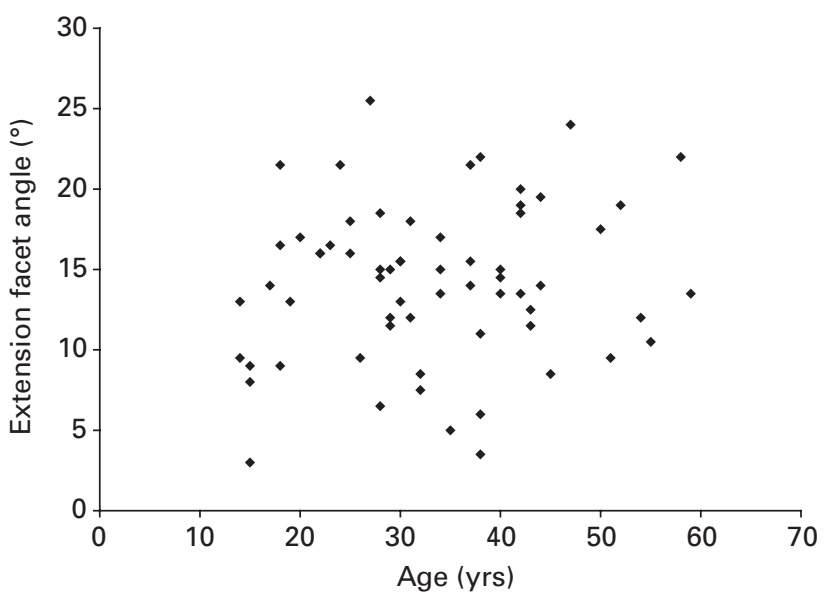

Fig. 3

Extension facet angle versus age in knees with acutely ruptured anterior cruciate ligaments serving as normal controls.

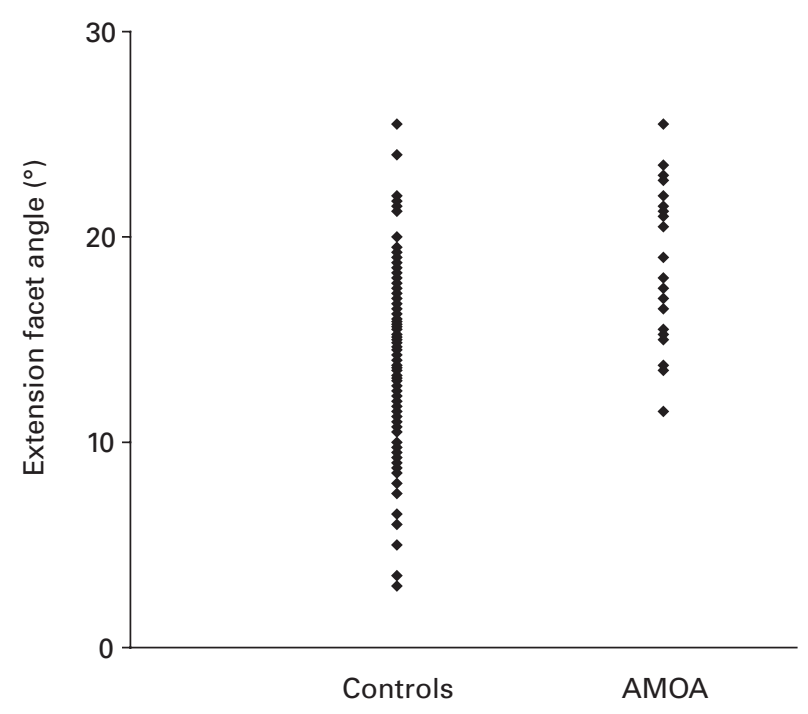

Fig. 4

Extension facet angle in normal controls with acutely ruptured anterior cruciate ligaments and patients with anteromedial osteoarthritis (AMOA).

HLC), but this was an outlier, all other measurements being within $4^{\circ}$.

The mean extension facet angle in patients with an isolated ACL rupture where there was no degeneration of the articular surface was $14^{\circ}\left(3^{\circ}\right.$ to $\left.25^{\circ}\right)$. The EFA was not related to age in this group (Fig. 3; Spearman's rank coefficient, $\mathrm{p}=0.30, \mathrm{r}=0.13)$. Men had a higher mean extension facet angle (men $15^{\circ}\left(3^{\circ}\right.$ to $\left.25^{\circ}\right)$, women $12^{\circ}\left(5^{\circ}\right.$ to $\left.19^{\circ}\right)$, Mann-Whitney $\mathrm{U}$ test, $\mathrm{p}=0.04)$.

The extension facet angle in the group with early anteromedial OA was a mean of $19^{\circ}\left(13^{\circ}\right.$ to $\left.26^{\circ}\right)$, significantly higher than in the patients with isolated tears of the ACL who served as a control group (Fig. 4; Mann-Whitney U test, $\mathrm{p}<0.001)$. A high angle $\left(\geq 20^{\circ}\right)$ was found in nine of 20 patients $(45 \%)$ in the anteromedial OA group, but only seven of 64 patients $(11 \%)$ in the ACL-ruptured control group. A low angle $\left(<10^{\circ}\right)$ was found in 14 of 64 patients $(22 \%)$ in the ACL-ruptured control group, but in none of the anteromedial OA group. As the anteromedial OA group had a higher proportion of women than the control group, the higher extension facet angle in the anteromedial OA group is unlikely to be a gender effect.

\section{Discussion}

This study demonstrates a wide variation in the anatomy of the anteromedial aspect of the tibial plateau in a group of patients with isolated acute ACL rupture, who provided the closest available approximation to normal knees for which MR scans were available. The mean extension facet angle in this group is higher than that reported by Pinskerova et $\mathrm{al}^{8}$ in a small group of cadaver knees, although their measurements were in the same range. The extension facet angle is slightly higher in men than in women, this was statistically significantly $(\mathrm{p}=0.04)$, and is not related to age. The method of measurement was equivalent to that used previously $^{7,8}$ and has been shown to be reproducible.

Although it is possible that the onset of degenerative changes at the base of the extension facet could result in an increase in the extension facet angle, we do not believe that this is the reason for the higher observed angles in the anteromedial OA group, as the angle was measured at the junction between cartilage and subchondral bone, and only using scans with early non-erosive degenerative changes. Instead, we postulate that there may be a causal link between a high extension facet angle and the onset of anteromedial OA, although we acknowledge that this is not proven by the association shown in this study. We have two theories, which we have not verified experimentally, that may explain this. Both assume that the shape of the femur, specifically the magnitude of the kink angle, ${ }^{9}$ varies independently of the extension facet angle. We have remeasured the kink angles and the extension facet angles in the six cadaver knees reported previously ${ }^{8}$ and found that in this limited sample there was no relation between the two.

The first theory rests on the assumption that the greater the extension facet angle, the sooner the tibial extension facet will contact the femur as the knee extends. For example, a tibial extension facet angled at $25^{\circ}$ might contact the femur at $25^{\circ}$ flexion, whereas one angled at $10^{\circ}$ might not contact it until $10^{\circ}$. If this is so, information derived from gait analyses ${ }^{10}$ shows that the steeper extension facet would be in contact almost throughout the stance phase of level walking, whereas the flatter extension facet would be in contact for only $50 \%$ of the stance phase. Doubling the duration of loaded contact for each step might increase the likelihood of cartilage damage. The second theory rests on 
the assumption that the ACL is under tension towards full extension, and that this tensile force is reacted to by a compressive force, in addition to those forces created by body weight and muscle action, on the articular cartilage in contact at the extension facet. If this is true, the steeper the tibial extension facet, the greater this additional force will be. Therefore, again, a steep extension facet might lead to articular cartilage damage.

This study was based on a group with an MRI diagnosis of early degenerative change in the medial compartment. The symptoms which led to the MRI request and the initial diagnosis made by the clinician are not known. Now that the variation in normal extension facet angle has been defined in our series, further work is needed to analyse patients with clinically apparent anteromedial OA to see whether their medial tibial plateau anatomy corresponds to this pattern, and whether or not this has any relationship with their femoral kink angle.

The outcome for those patients in the control group with isolated ACL rupture and a high extension facet angle but no evidence of OA is not known. A prospective analysis would be required in this group, with appropriate ethical approval.

The possibility of identifying a group of patients with symptomatic early anteromedial OA (with MRI and arthroscopic confirmation) raises the prospect of performing an excision osteotomy to reduce the extension facet angle by removing a small wedge of bone from beneath the anteromedial tibial plateau.

The authors would like to thank Dr Hazel Taylor from the Research and Development Unit at the Bristol Royal Infirmary for assistance with study design and statistical analysis and Dr Charles Wakeley, Consultant Radiologist at the Bristol Royal Infirmary, for helpful discussion.

No benefits in any form have been received or will be received from a commercial party related directly or indirectly to the subject of this article.

\section{References}

1. Ahlbäck S. Osteoarthritis of the knee: a radiographic investigation. Acta Radiol Diagn (Suppl) 1968;277:7-72.

2. White SH, Ludkowski PF, Goodfellow JW. Anteromedial osteoarthritis of the knee. J Bone Joint Surg [Br] 1991;73-B:582-6.

3. Keyes GW, Carr AJ, Miller RK, Goodfellow JW. The radiographic classification of medial gonarthritis: correlation with operation methods in 200 knees. Acta Orthop Scand 1992;63:497-501.

4. Harman MK, Markovich GD, Banks SA, Hodge WA. Wear patterns on tibial plateaus from varus and valgus osteoarthritic knees. Clin Orthop 1998;352:149-58.

5. Moschella D, Blasi A, Leardini A, Ensini A, Catani F. Wear patterns on tibial plateau from varus osteoarthritic knees. Clin Biomech (Bristol, Avon) 2006;21:152-8.

6. Pinskerova V, Maquet P, Freeman MAR. Writings on the knee between 1836 and 1917. J Bone Joint Surg [Br]2000;81-B:1100-2.

7. Iwaki H, Pinskerova V, Freeman M. Tibiofemoral movement. 1: the shapes and relative movements of the femur and tibia in the unloaded cadaver knee. J Bone Joint Surg [Br] 2000;82-B:1189-95.

8. Pinskerova V, Iwaki H, Freeman $\mathbf{M}$. The shapes and relative movements of the femur and tibia in the unloaded cadaveric knee: a study using MRI as an anatomic tool. In: Insall JN, Scott R, eds. Third ed. Surgery of the knee. Philadelphia: WB Saunders, 2001.

9. Albrecht H. Zur anatomie des kniegelenkes. In: Diss. Deutsche Zeits u Chirurgie, 1876:433.

10. Lafortune MA, Cavanagh PR, Sommer HJ, Kalenak A. Three dimensional kinematics of the human knee during walking. J Biomech 1992;25:347-57. 